\title{
Floquet's Unit Cell Design for Periodic Structures at Optical Frequencies
}

\author{
Alessandro Massaro, ${ }^{1}$ Roberto Cingolani, ${ }^{1}$ Adriana Passaseo, ${ }^{1,2}$ and Massimo De Vittorio ${ }^{1}$ \\ ${ }^{1}$ National Nanotechnology Laboratory of CNR-INFM, Università del Salento, Distretto Tecnologico-ISUFI, \\ Via Arnesano, 73100 Lecce, Italy \\ ${ }^{2}$ IMM-CNR Sezione Lecce, University Campus, Lecce-Monteroni, 73100 Lecce, Italy
}

Correspondence should be addressed to Alessandro Massaro, massaroale@libero.it

Received 25 March 2009; Accepted 7 July 2009

Recommended by Kamya Yekeh Yazdandoost

\begin{abstract}
We present a new theoretical approach regarding the design of 2D periodic structure at optical frequencies. The model is based on Floquet's theory and on the variational equivalent circuit. The distributed circuit model is developed through the use of the microwave network theory and the optical theory of the step discontinuities. This approach analyzes $2 \mathrm{D}$ dielectric periodic structures with high dielectric contrast by the transmission line model including variational equivalent circuits. The 3D Finite Element Method (FEM) model validates Floquet's design theory of the grating resonance and provides the design optimization of an optical GaAs periodic waveguide.
\end{abstract}

Copyright ( $(2009$ Alessandro Massaro et al. This is an open access article distributed under the Creative Commons Attribution License, which permits unrestricted use, distribution, and reproduction in any medium, provided the original work is properly cited.

\section{Introduction}

Various scientific and engineering applications [1-11] can be realized by utilizing dielectric periodic devices. Technological improvements for high frequency periodic structures are developed in order to fabricate photonic crystal structures for power dividers, filters [4-9], directional couplers, and wavelength division multiplexer $[10,11]$. New classes of dielectric materials which are often artificially fabricated for this purpose are referred as photonic crystals and often are characterized by high dielectric contrast. The effect of the high dielectric contrast, at high working frequencies, is relevant in the frequency response especially for discontinuous structures such as cascade of step discontinuities. Step discontinuities in planar dielectric waveguides are commonly used in integrated circuits ranging from submillimetre to optical frequencies. The step discontinuities in dielectric slab waveguides are, in fact, tailored for several components such as distributed feedback lasers, gratings, transformers, antenna feed, and others. It is thus important to have accurate and reliable theoretical prediction of the behavior of this discontinuity. Step discontinuity model, presented in this work, starts from the junction between two slabs of different heights as two lines with different impedances.
As a refinement of this model we introduce the variational equivalent circuit $[12,13]$ which minimizes the effect of the discontinuity for a periodic structure. By modeling a periodic dielectric structure as a cascade of unit cell elements [12-17] (see Figures 1 and 2(a)) the error of the frequency response of a single cell increases drastically for a long structure. Periodic structures with low dielectric contrast can be analyzed by the simplified transmission line models [16] reported in Figure 2(b), but, for high dielectric contrasts, an accurate modeling is requested. In this way it is necessary to introduce the transformation ratio that takes into account the amplitude difference of the traveling signals along the discontinuous waveguide. The transformation ratio, obtained through the modal analysis of the optical waveguide, allows to analyze high dielectric contrast waveguides such as air holes in GaAs material. This aspect is considered in the proposed theoretical approach with the equivalent variational circuit of a step discontinuity $[12,13]$. The frequency behavior of periodic structures at optical frequencies was analyzed in previous works $[18,19]$ by impedance matching technique. In the presented work an alternative and rigorous technique is combined with the Brillouin theory. This approach provides a useful new tool for photonic crystal design. 
In particular in the presented work the following aspects are investigated: (i) design of $2 \mathrm{D}$ periodic waveguides by distributed transmission line model at optical frequencies, including Floquet's theorem [15-17], Brillouin diagram [15], and equivalent variational circuit of the propagating modes [12]; (ii) analytical example of a periodic GaAs slab waveguide at working frequency $\lambda_{0}=1.55 \mu \mathrm{m}$; (iii) $3 \mathrm{D}$ finite element method (FEM) simulation of a $3 \mathrm{D}$ GaAs periodic structure by analyzing the effect of the 3D cell shape on the frequency response with respect to the $2 \mathrm{D}$ theoretical approach.

\section{Floquet's Theory}

The periodic structure of Figure 1(a) is analyzed by considering the study of the resonance in the propagating directions. The theoretical model considers the propagation characteristic of the infinite loaded line as cascade of unit cells shown in Figure 1(b). Each unit cell of this line consists of transmission lines with length $L_{1}$ and $L_{2}$ which represent the planar dielectric waveguide with high and low refractive index, respectively, and of two ideal transformers which models the step discontinuities at optical frequencies. The wave-amplitude transmission matrix $(\mathrm{ABCD})$ is used for the unit cell of Figure 1, where

$$
\left(\begin{array}{l}
V_{1} \\
V_{2}
\end{array}\right)=\left(\begin{array}{ll}
A & B \\
C & D
\end{array}\right)_{u} \cdot\left(\begin{array}{l}
I_{1} \\
I_{2}
\end{array}\right),
$$

where $u$ indicates the unit cell transmission matrix which includes the step discontinuity, and is expressed as

$$
\begin{aligned}
& \left(\begin{array}{ll}
A & B \\
C & D
\end{array}\right)_{u}=\left(\begin{array}{cc}
\cos \left(\theta^{\prime}\right) & i Z_{0}^{\prime} \sin \left(\theta^{\prime}\right) \\
\frac{i \sin \left(\theta^{\prime}\right)}{Z_{0}^{\prime}} & \cos \left(\theta^{\prime}\right)
\end{array}\right) \cdot\left(\begin{array}{ll}
r & 0 \\
0 & \frac{1}{r}
\end{array}\right) \\
& \cdot\left(\begin{array}{cc}
\cos (\theta) & i Z_{0} \sin (\theta) \\
\frac{i \sin (\theta)}{Z_{0}} & \cos (\theta)
\end{array}\right) \cdot\left(\begin{array}{cc}
\frac{1}{r} & 0 \\
0 & r
\end{array}\right) \\
& \cdot\left(\begin{array}{cc}
\cos \left(\theta^{\prime}\right) & i Z_{0}^{\prime} \sin \left(\theta^{\prime}\right) \\
\frac{i \sin \left(\theta^{\prime}\right)}{Z_{0}^{\prime}} & \cos \left(\theta^{\prime}\right)
\end{array}\right),
\end{aligned}
$$

where $r$ is the transformation ratio. If the periodic structure is capable of supporting a propagating wave, it is necessary for the voltage and current at the $(m+1)$ th cell terminal [1215 ] to be equal to the voltage and current at the $m$ th terminal, apart from a phase delay due to a finite propagation time. Hence, let us assume that

$$
V_{m+1}=V_{m} e^{-\gamma l}, \quad I_{m+1}=I_{m} e^{-\gamma l},
$$

where $\gamma=\alpha+i \beta_{z}$ is the propagation constant along the $z$ propagating direction of the periodic structure, and $l=\Lambda=$ $2 L_{1}+L_{2}$ is the unit-cell length. For a Bloch wave [12], the eigenvalue equation is given by

$$
A D+e^{2 \gamma l}-(A+D) e^{\gamma l}-B C=0 .
$$

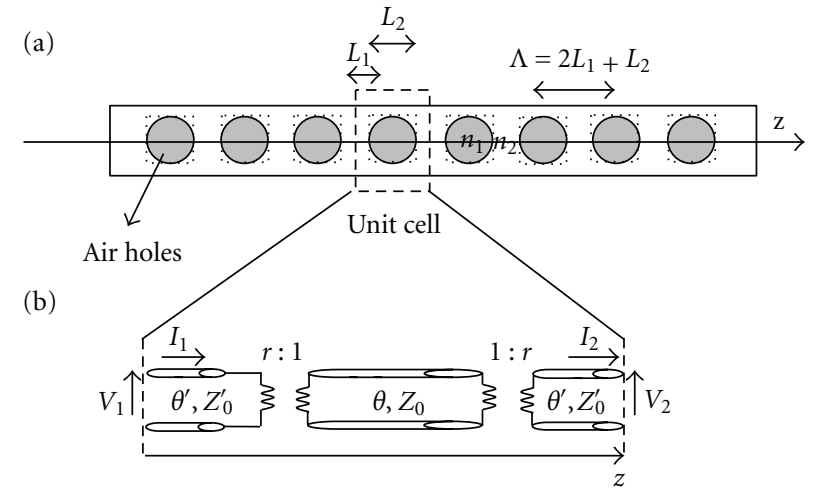

Figure 1: (a) Periodic structure with air holes in dielectric material; (b) equivalent $1 \mathrm{D}$ transmission line of the unit cell: $Z_{0}$ and $Z_{0}^{\prime}$ are the characteristic impedances and $\theta^{\prime}=\beta_{z}^{\prime} L_{1}$ and $\theta=\beta_{z} L_{2}$ are the electrical lengths.

Being the determinant of the unit cell transmission matrix equal to 1 , then (4) becomes

$$
\cosh (\gamma l)=\frac{A+D}{2} .
$$

In the hypothesis of propagation along one direction $\left(\beta_{z} \neq 0\right)$ without losses $(\alpha=0)$, then (5) represents the following Floquet's condition:

$$
\frac{A+D}{2} \leq 1,
$$

which indicates the passband region of the periodic structure. For a cascade of unit cells the total ABCD matrix refers to the product of all the unit cell transmission matrices.

\section{Brillouin Diagrams and 2D Model}

When studying the passband and the stopband characteristic of a periodic structure, it is useful to plot the propagation constant $\beta_{z}$ versus the propagation constant $n_{2} k_{0}$ of the unloaded line (uniform planar waveguide), where $k_{0}$ is the free space wavelength. Such a graph is called Brillouin [15] diagram. The diagram plots the dispersion relation for the planar waveguide mode

$$
\beta_{z}=\sqrt{\left(n_{2} k_{0}\right)^{2}-k_{c}^{2}}
$$

or

$$
n_{2} k_{0}=\sqrt{\beta_{z}^{2}+k_{c}^{2}},
$$

where $n_{2}$ is the refractive index of the core planar waveguide, and $k_{c}$ is the cutoff wavenumber of the propagating mode. The Brillouin diagram is also useful in order to interpret the various wave velocities associated with a dispersive structure. In particular it is possible to evaluate the phase velocity

$$
v_{p}=\frac{\omega}{\beta_{z}}
$$




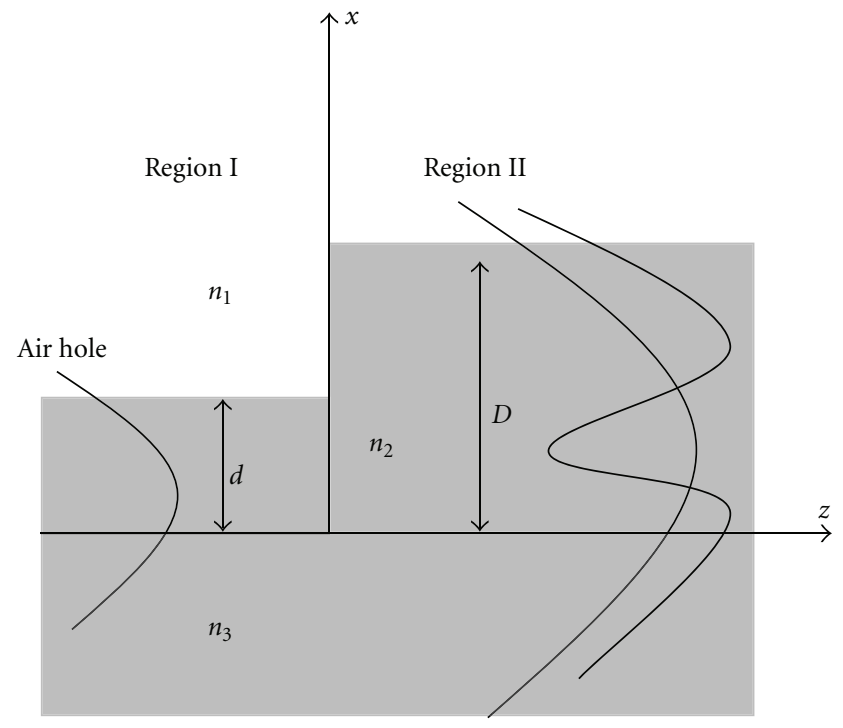

(a)

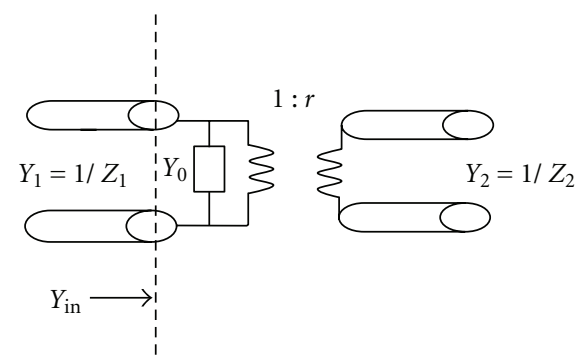

(c)

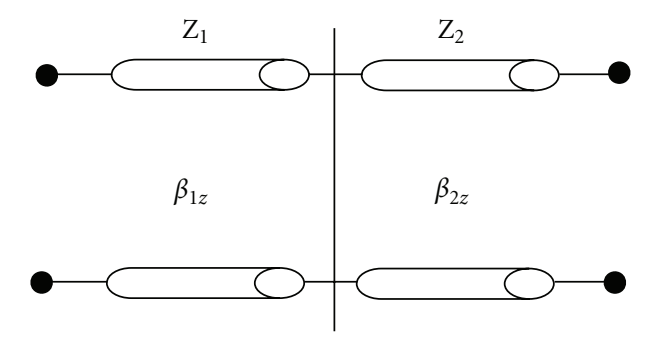

(b)

FIGURE 2: (a) Step discontinuity: junction between two dielectric slabs with core thicknesses $d$ and $D$ (longitudinal section of the unit cell shown in Figure 1(a)). (b) Simplified equivalent circuit for small step discontinuity and low dielectric contrast $\Delta n=n_{2}-n_{1}$. (c) Variational equivalent circuit for a step discontinuity. (d) Example of variational equivalent circuit of a double step discontinuity with one guided mode in region I and two guided modes in region II.

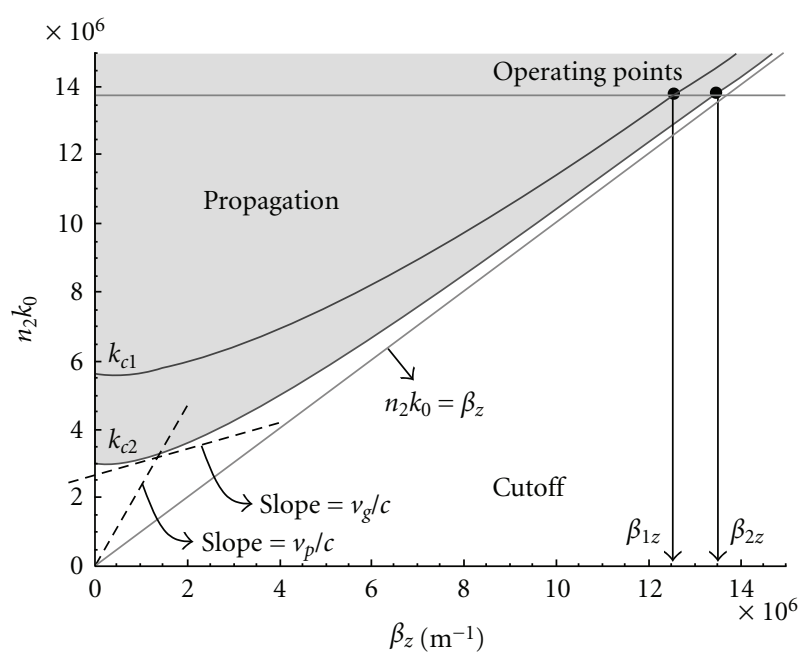

FIgure 3: Brillouin diagram for a GaAs $\left(n_{2}=3.408\right)$ junction on a substrate with $n_{3}=3.1$. The operating points refer to a working wavelength $\lambda_{0}=1.55 \mu \mathrm{m} ; \beta_{1 z}$ and $\beta_{2 z}$ are the effective propagation constants along the $z$-propagating direction for the fundamental TE modes in regions I and II of Figure 2(a), respectively; $k_{c 1}$ and $k_{c 2}$ are the cutoff wavenumbers. and the group velocity

$$
v_{g}=\frac{d \omega}{d \beta_{z}} .
$$

For a generic $3 \mathrm{D}$ case the Brillouin diagram takes into account all the propagation constants in the $x, y, z$ directions by the wavenumber conservation law [12]

$$
n_{2} k_{0}=\sqrt{\beta_{z}^{2}+\beta_{x}^{2}+\beta_{y}^{2}} .
$$

The conservation law (11) applied to a bidimensional waveguide, and Floquet's condition (5) allow to define the unit cell dimensions for a 2D periodic pattern [16].

\section{Step Discontinuity: Variational Equivalent Circuit}

Periodic air holes in GaAs material can be modeled by the analogy with the cascade step discontinuities. A simple and effective equivalent circuit for the small step (dielectric junctions) between two slabs waveguide is shown in Figure 2. The single step discontinuity of a planar waveguide is reported 


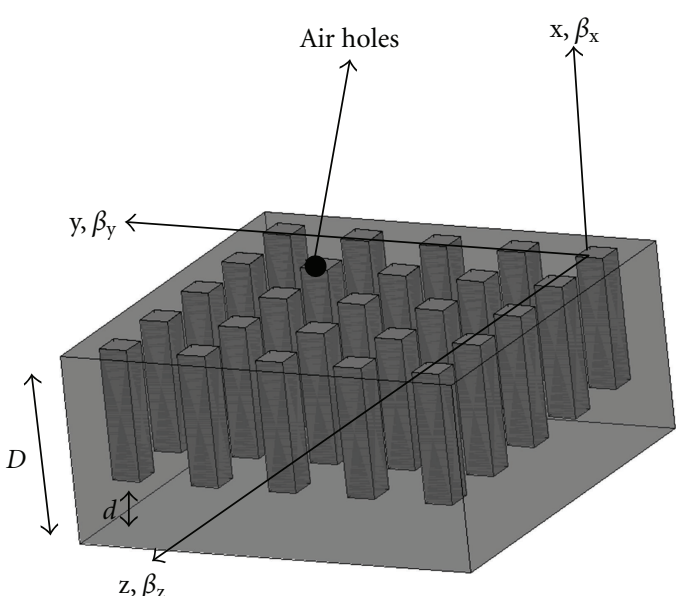

(a)

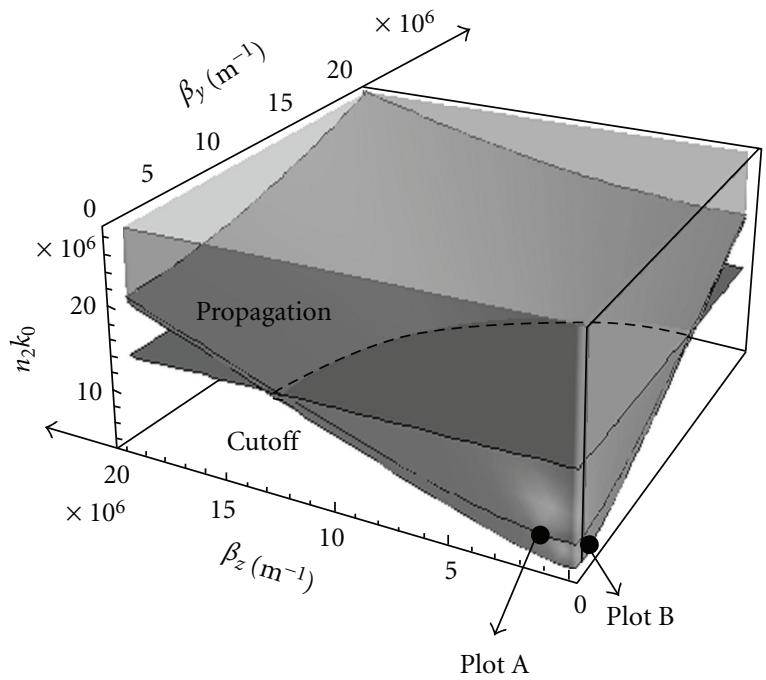

(b)

FIgURE 4: Brillouin diagram for 3D GaAs periodic structures with air holes and different periodicity along the $y$-and $z$-axis. (a) 3D periodic structure; (b) Brillouin diagram: the dashed line represents the set of operating points at working wavelength $\lambda_{0}=1.55 \mu \mathrm{m}$; plot A refers to the waveguide with core thickness $d$; plot B refers to the waveguide with core thickness $D$.

in Figure 2(a) where $d$ and $D$ are the core thicknesses of the two waveguides. In Figures 2(b) and 2(c) are reported the simple equivalent circuit for a small step discontinuity (low dielectric contrast or small difference between $D$ and $d$ ) and for the variational equivalent circuit, respectively. If more modes are involved, the generic variational equivalent circuit considers each line for each propagating mode. In particular in Figure 2(d) is shown the case of two guided modes in the region with $D$ core thickness, for a double step discontinuity. For The transverse electric (TE) mode excitation defines the following three field components:

$$
H_{x}(x, z)=\frac{1}{i \omega \mu} \frac{\partial}{\partial z} E_{y}(x, z), \quad H_{z}(x, z)=-\frac{1}{i \omega \mu} \frac{\partial}{\partial x} E_{y}(x, z),
$$

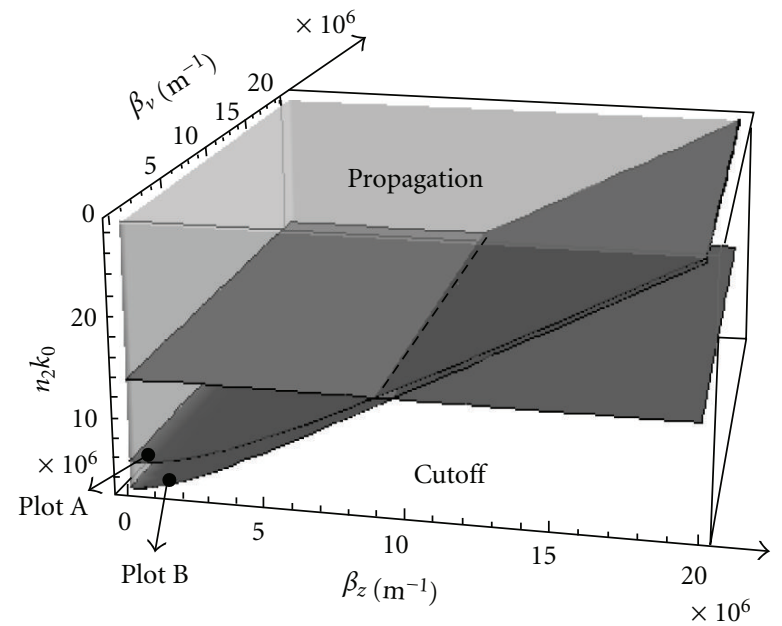

FIGURE 5: Brillouin diagram for 3D GaAs periodic structures with air holes and same periodicity along the $y$ - and $z$-axis. The dashed line represents the set of operating points at working wavelength $\lambda_{0}=1.55 \mu \mathrm{m}$; plot A refers to the waveguide with core thickness $d$; plot $\mathrm{B}$ refers to the waveguide with core thickness $D$.

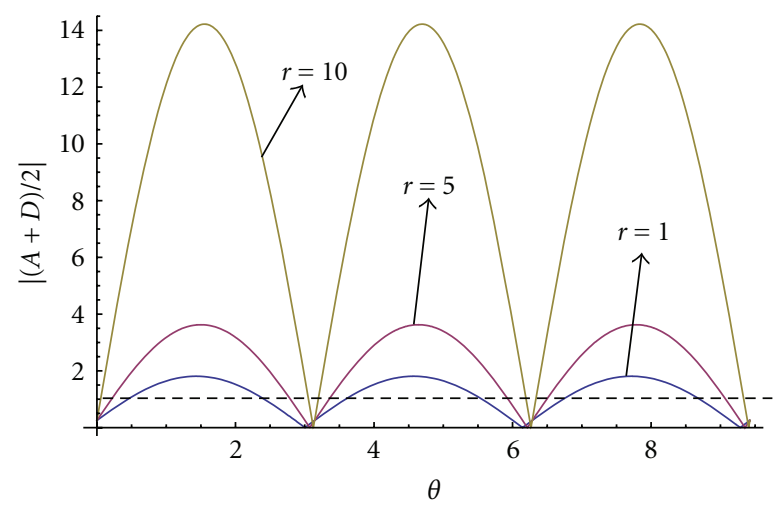

FIGURE 6: Single unit cell Floquet's resonance condition (passband condition) for different ratio transformation $r$. The passband condition is below the dashed line: in this case, $n_{2}=3.408, L_{1}=$ $1 \mu \mathrm{m}, \lambda_{0}=1.55 \mu \mathrm{m}$, and $L_{2}$ can be fixed in order to select the resonance condition.

where $E_{y}(x, z)$ is represented by the modal expansion [12]

$$
E_{y}(x, z)=\left\{\sum_{k} a_{k} \psi_{k}(x)+\int_{0}^{\infty} b\left(k_{x}\right) \phi\left(x ; k_{x}\right) d k_{x}\right\} e^{-i \beta_{z} z}
$$

where $a_{k}$ and $b\left(k_{x}\right)$ are the amplitudes of the guided and continuum modes, respectively. For pronounced discontinuities, a considerable mode mixing characterizes the junction. An effective way to consider such mode mixing is to derive an equivalent circuit by using a variational approach $[12,13]$. Let us indicate by $\psi_{k}$ the guided TE wave and by $\phi$ the mode functions pertaining to the continuous spectrum in regions 


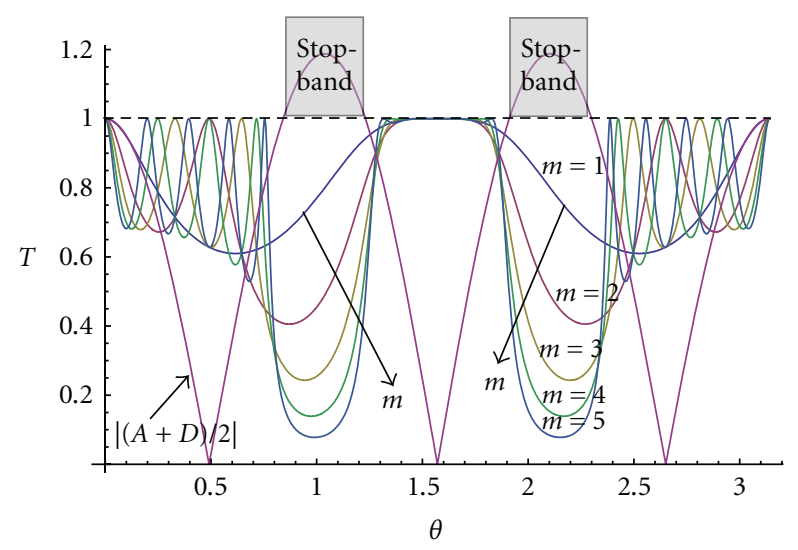

FIGURE 7: Floquet's resonance condition (passband condition) for different unit cell numbers $m$. The passband condition is below the dashed line: in this case, $r=2, Z_{0}^{\prime}=2, Z_{0}=1$, and $\theta^{\prime}=\theta$.

I and II of Figure 2(a), respectively. By using (13) the field at the left and at the right of the discontinuity is the following:

$$
\begin{aligned}
E_{\text {left }} & =(1+R) \sum_{k} a_{k} \psi_{k}(x)+\int_{0}^{\infty} A\left(k_{x}\right) \phi\left(x ; k_{x}\right) d k_{x}, \\
E_{\text {right }} & =T \sum_{k^{\prime}} a_{k^{\prime}}^{\prime} \psi_{k^{\prime}}^{\prime}(x)+\int_{0}^{\infty} B\left(k_{x}\right) \phi^{\prime}\left(x ; k_{x}^{\prime}\right) d k_{x}^{\prime},
\end{aligned}
$$

where $R$ indicates the reflection coefficient, and $T$ is the transmission coefficient. The orthonormality property of the modes provides the following relationships:

$$
\begin{aligned}
1+R & =\int_{-\infty}^{+\infty} E(x) \sum_{k} \psi_{k}\left(x ; k_{x}\right) d x, \\
A\left(k_{x}\right) & =\int_{-\infty}^{+\infty} E(x) \phi_{k}\left(x ; k_{x}\right) d x, \\
T & =\int_{-\infty}^{+\infty} E(x) \sum_{k^{\prime}} \psi_{k^{\prime}}^{\prime}\left(x ; k_{x}^{\prime}\right) d x, \\
B\left(k_{x}^{\prime}\right) & =\int_{-\infty}^{+\infty} E(x) \phi_{k^{\prime}}\left(x ; k_{x}^{\prime}\right) d x .
\end{aligned}
$$

However, the transverse magnetic field at each side of the discontinuity must be continuous, thus requiring

$$
\begin{gathered}
(1-R) \sum_{k} Y_{1 k} \psi_{k}(x)-\int_{0}^{\infty} Y_{1 k}\left(k_{x}\right) A\left(k_{x}\right) \phi_{k}\left(x ; k_{x}\right) d k_{x} \\
=T \sum_{k^{\prime}} Y_{2 k^{\prime}} \psi_{k^{\prime}}^{\prime}(x)+\int_{0}^{\infty} B\left(k_{x}^{\prime}\right) \phi_{k^{\prime}}^{\prime}\left(x ; k_{x}^{\prime}\right) d k_{x}^{\prime},
\end{gathered}
$$

where $Y_{1 k}$ and $Y_{2 k^{\prime}}$ are the characteristic modal admittances of the transmission lines related to regions I and II, respectively, (see Figure 2(c)). Let us assume for simplicity that only the guided mode $\Psi_{1}$ exists in region $\mathrm{I}$, and $\Psi_{2}$ is present in region II. Let us also indicate by $\phi_{1}, \phi_{2}$ the mode functions pertaining to the continuous spectrum of regions
I and II of Figure 2(a). By multiplying (16) by $E$ (electric field at junction), by integrating over $x$, and by dividing by $(1+R)^{2}$ it is possible to evaluate the input admittance of Figure 2(c) as

$$
\begin{aligned}
Y_{\text {in }}= & \frac{1-R}{1+R}=\frac{Y_{2}}{Y_{1}}\left[\frac{\int_{-\infty}^{+\infty} E(x) \psi_{2}\left(x ; k_{x}^{\prime}\right) d x}{\int_{-\infty}^{+\infty} E(x) \psi_{1}\left(x ; k_{x}\right) d x}\right]^{2} \\
& +\frac{1}{Y_{1}} \frac{\int_{0}^{\infty} Y_{1}\left(k_{x}\right)\left[\int_{-\infty}^{+\infty} E(x) \phi_{1}\left(x ; k_{x}\right) d x\right]^{2} d k_{x}}{\left[\int_{-\infty}^{+\infty} E(x) \psi_{1}\left(x ; k_{x}\right) d x\right]^{2}} \\
& +\frac{1}{Y_{1}} \frac{\int_{0}^{\infty} Y_{2}\left(k_{x}^{\prime}\right)\left[\int_{-\infty}^{+\infty} E(x) \phi_{2}\left(x ; k_{x}^{\prime}\right) d x\right]^{2} d k_{x}^{\prime}}{\left[\int_{-\infty}^{+\infty} E(x) \psi_{1}\left(x ; k_{x}\right) d x\right]^{2}}
\end{aligned}
$$

which can be rewritten as

$$
Y_{\text {in }}=r^{2} \frac{Y_{2}}{Y_{1}}+\frac{Y_{0}}{Y_{1}}
$$

with

$$
\begin{aligned}
r^{2}= & {\left[\frac{\int_{-\infty}^{+\infty} E(x) \psi_{2}\left(x ; k_{x}^{\prime}\right) d x}{\int_{-\infty}^{+\infty} E(x) \psi_{1}\left(x ; k_{x}\right) d x}\right]^{2}, } \\
Y_{0}= & \frac{\int_{0}^{\infty} Y_{1}\left(k_{x}\right)\left[\int_{-\infty}^{+\infty} E(x) \phi_{1}\left(x ; k_{x}\right) d x\right]^{2} d k_{x}}{\left[\int_{-\infty}^{+\infty} E(x) \psi_{1}\left(x ; k_{x}\right) d x\right]^{2}} \\
& +\frac{\int_{0}^{\infty} Y_{2}\left(k_{x}\right)\left[\int_{-\infty}^{+\infty} E(x) \phi_{2}\left(x ; k_{x}^{\prime}\right) d x\right]^{2} d k_{x}^{\prime}}{\left[\int_{-\infty}^{+\infty} E(x) \psi_{1}\left(x ; k_{x}\right) d x\right]^{2}}
\end{aligned}
$$

suggesting the equivalent variation circuit of Figure 2(c). It is noted that in this circuit the admittance $Y_{0}$ includes radiation and reactive effects of the modes which cannot propagate. By considering more propagating modes (19) becomes

$$
r=\frac{\int_{-\infty}^{+\infty} E(x) \sum_{k^{\prime}} a_{k^{\prime}}^{\prime} \psi_{k^{\prime}}^{\prime}(x) d x}{\int_{-\infty}^{+\infty} E(x) \sum_{k} a_{k} \psi_{k}(x) d x}
$$

Equation (21) shows that the generic ratio transformation is a function of the propagating modes. Moreover, the impedances characteristics of the transmission lines related to the regions I and II of Figure 2(a) are functions of the effective refractive indices [12], in particular

$$
Z_{1,2}=\frac{1}{n_{\mathrm{eI}, \mathrm{eII}}} \sqrt{\frac{\mu_{0}}{\varepsilon_{0}}}
$$

where $n_{\mathrm{eI}}$ and $n_{\mathrm{eII}}$ are the effective refractive index of the region I and region II, respectively. 


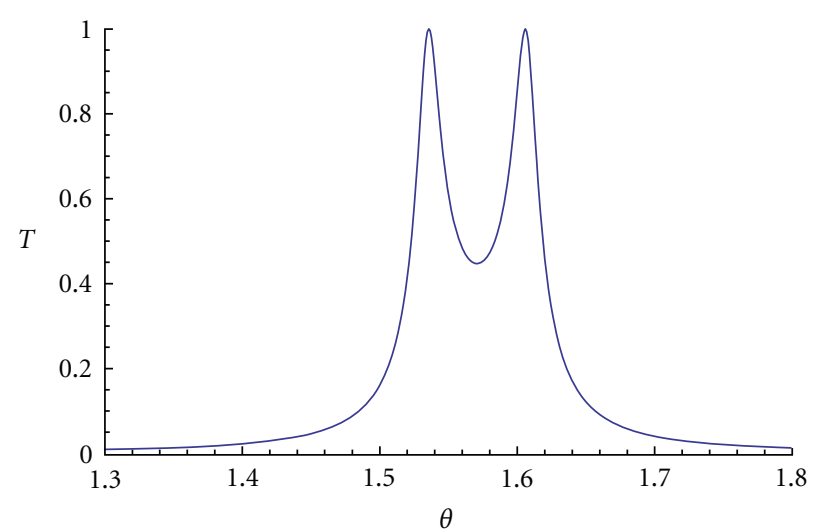

(a)

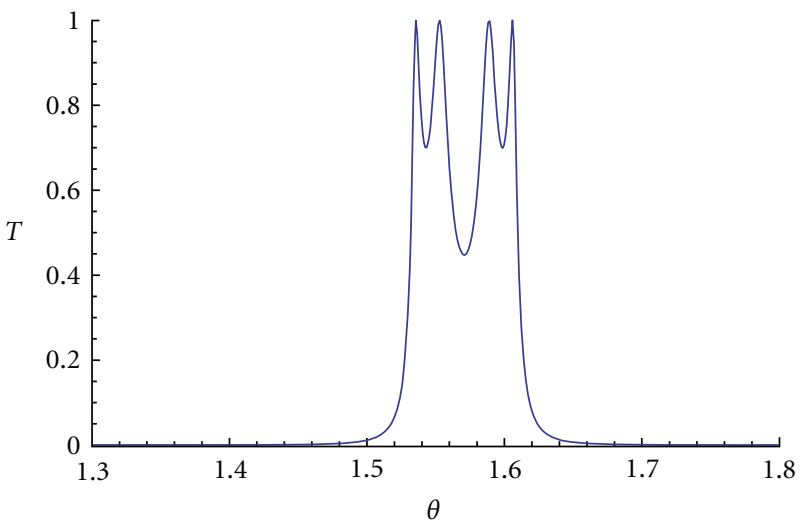

(c)

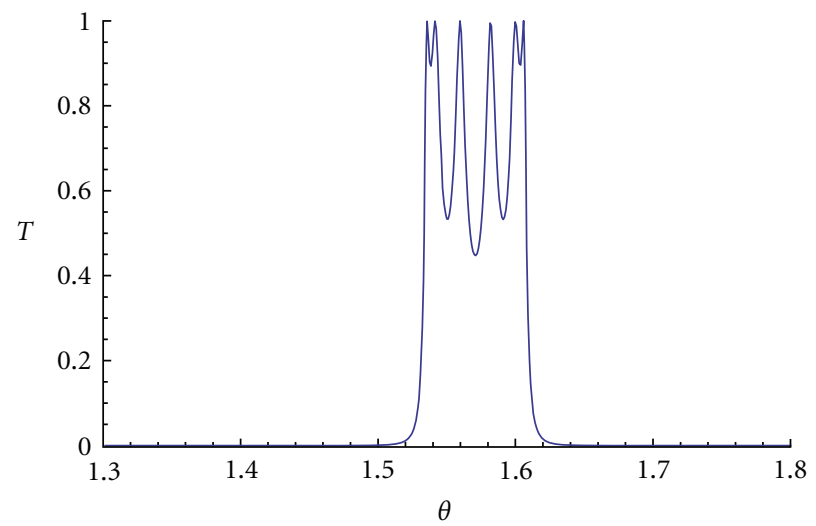

(e)

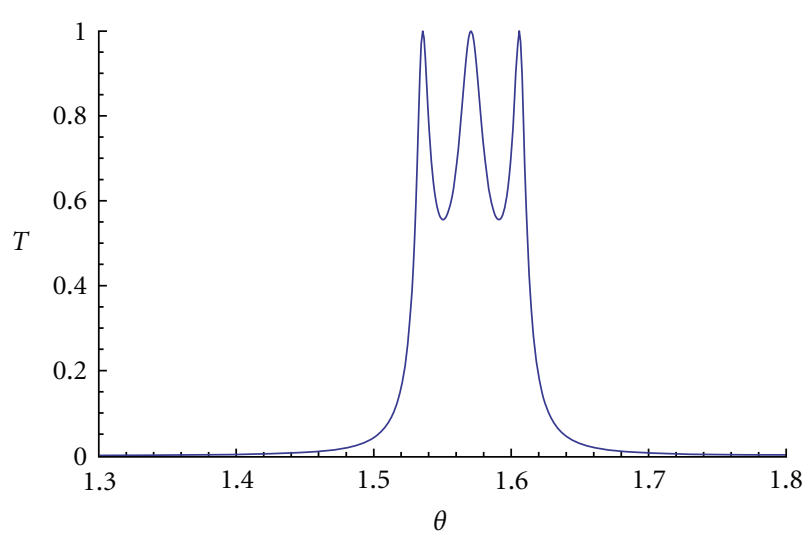

(b)

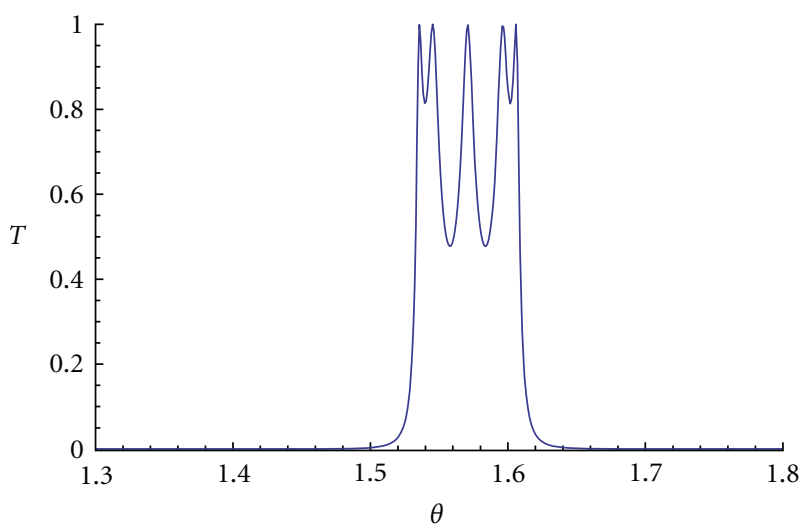

(d)

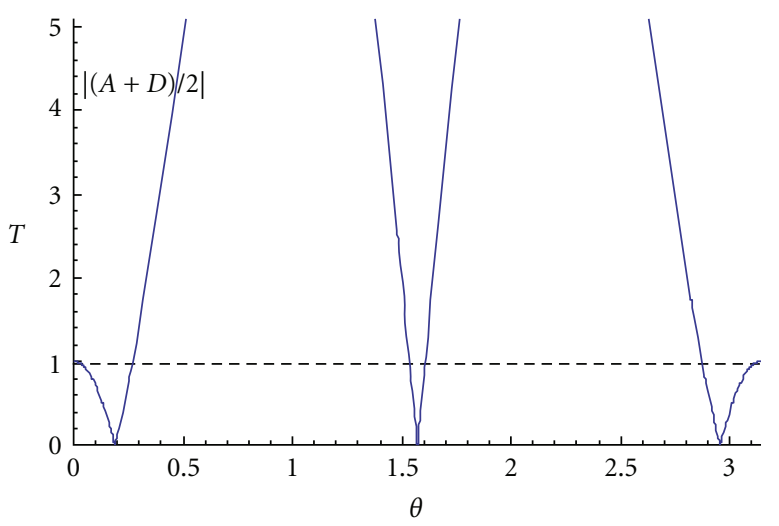

(f)

Figure 8: $1 \mathrm{D}$ practical case of GaAs $\left(n_{2}=3.408\right)$ slab waveguide with $L_{1}=0.1163 \mu \mathrm{m}, L_{2}=0.1246 \mu \mathrm{m}$ at central wavelength (resonance wavelength) and $\lambda_{0}=1.55 \mu \mathrm{m}$. (a) Transmittivity of 1 unit cell, (b) transmittivity of 2 unit cells, (c) transmittivity of 3 unit cells, (d) transmittivity of 4 unit cells, (e) transmittivity of 5 unit cells, and (f) Floquet's condition for 1 unit cell.

The TE modal profile reported by (19) and (21) is given by $[12]$

$$
\psi(x)= \begin{cases}a_{k} e^{-p(x-d)}, & \mathrm{x} \geq d, \\ a_{k} \frac{\cos \left(k_{x} x-\bar{\varphi}\right)}{\cos \left(k_{x} d-\bar{\varphi}\right)}, & 0 \leq \mathrm{x} \leq d, \\ a_{k} \frac{\cos (\bar{\varphi})}{\cos \left(k_{x} d-\bar{\varphi}\right)} e^{q x}, & \mathrm{x} \leq 0,\end{cases}
$$

$$
\begin{aligned}
\bar{\varphi} & =\tan ^{-1}\left(\frac{q}{k_{x}}\right), \\
a_{k} & =\sqrt{\frac{2}{1 / p+d+1 / q}} \cos \left(k_{x} d-\bar{\varphi}\right),
\end{aligned}
$$

where $p, q$, and $k_{x}$ are the $x$-transverse propagation (see Figure 2(a)) constant in the cladding, in the core and in 


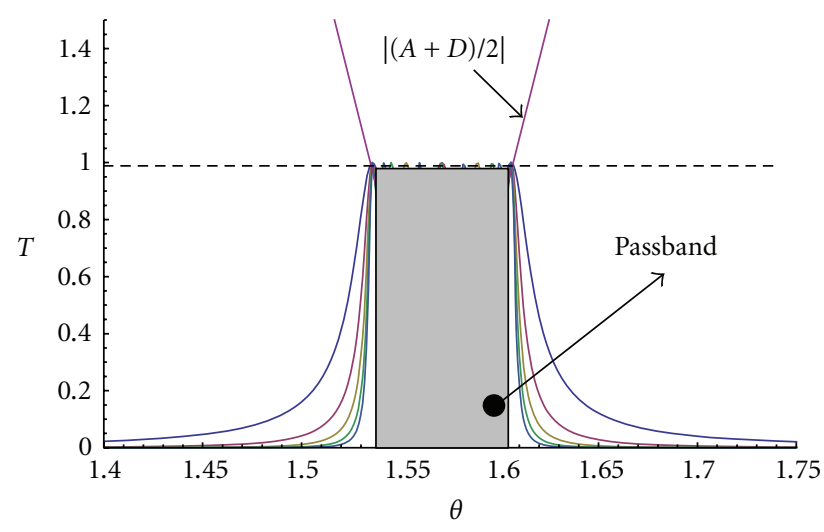

(a)

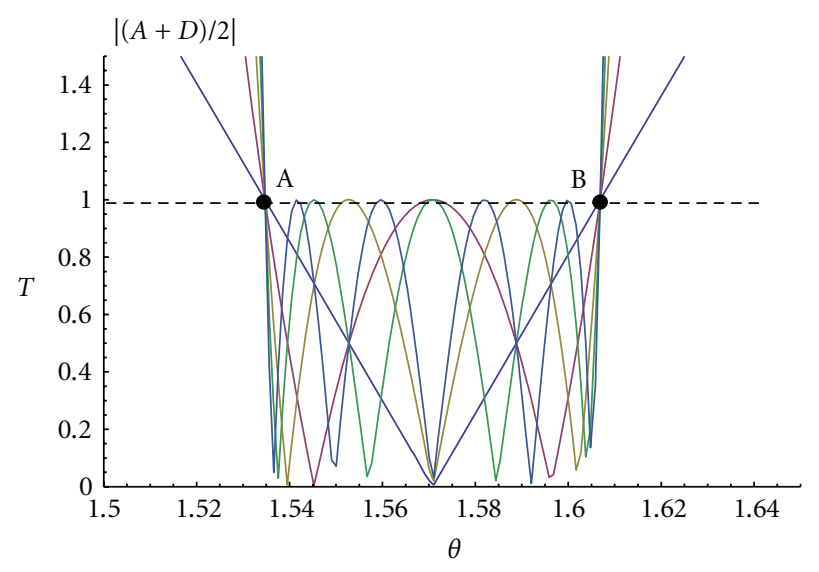

(b)

Figure 9: (a) Figures 8(a), 8(b), 8(c), 8(d), 8(e), and 8(f) on the same graph. (b) Floquet's conditions for different unit cells number.

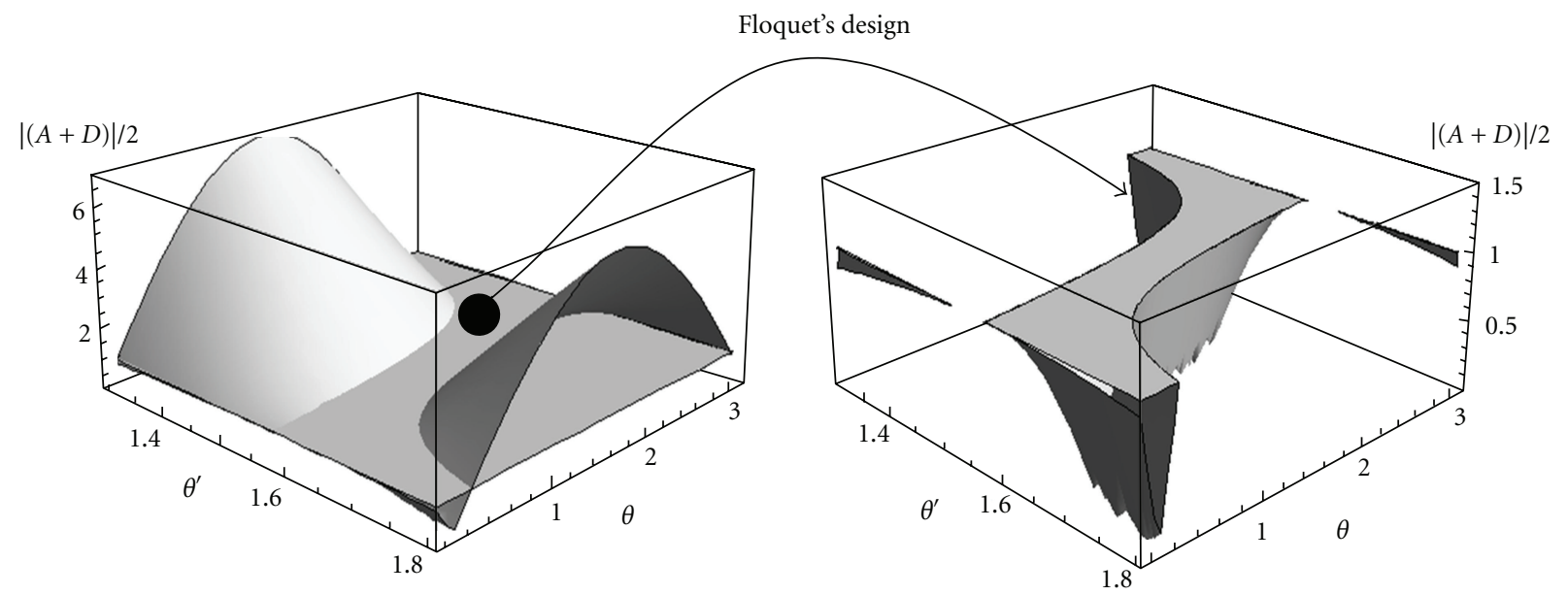

(a)

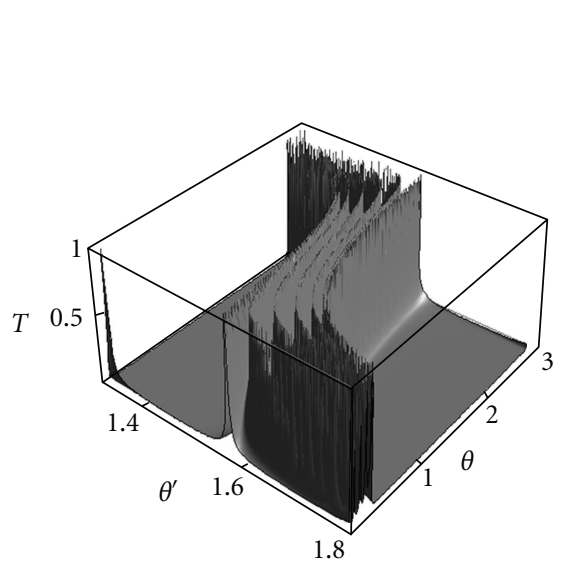

(b)

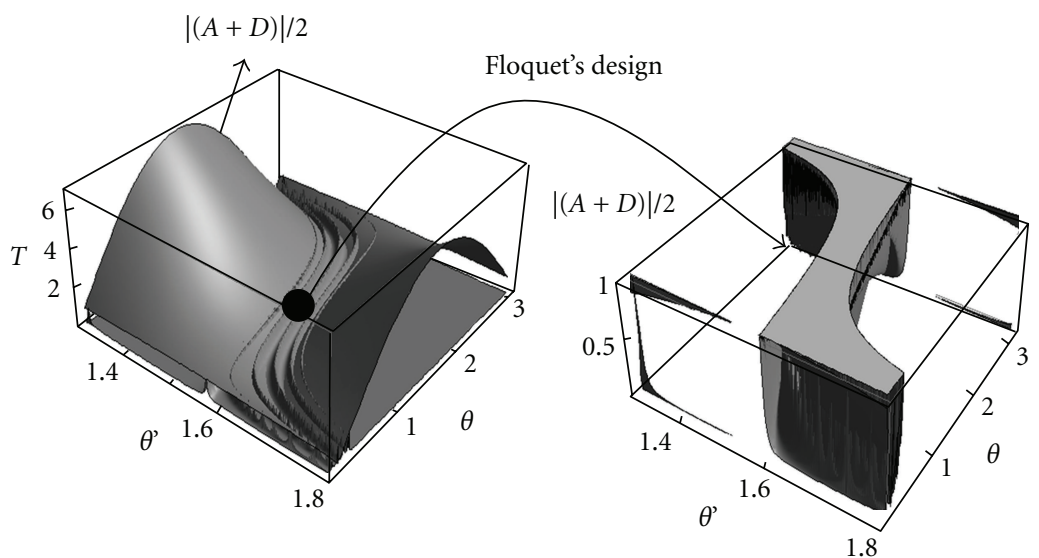

(c)

Figure 10: (a) Floquet's region $|A+D| / 2<1$ for a single unit cell. (b) Floquet's region for five unit cells. 
the substrate, respectively. The TE modal profiles $\Psi^{\prime}$ are obtained from (22) by substituting $k_{x}^{\prime}$ to $k_{x}, p^{\prime}$ to $p, q^{\prime}$ to $q$, and $a_{k}^{\prime}$ to $a_{k}$ and $d$ to $D$. The same model can be applied also to transverse magnetic (TM) fields.

\section{Analytical Results: Floquet's Design}

As example an asymmetrical slab waveguide (Figure 2(a)) with $n_{1}=1, n_{2}=3.408, n_{3}=3.1, d=0.24 \mu \mathrm{m}, D=0.8 \mu \mathrm{m}$ is analyzed. By supposing a wavelength grating resonance at $\lambda_{0}=1.55 \mu \mathrm{m}$, the Brillouin diagram of Figure 3 is obtained. In this diagram are considered the fundamental TE modes in regions I and II of Figure 2(a). The evaluated propagation constant along the $z$-direction is $\beta_{z 1}=1.2625 * 10^{7} \mathrm{~m}^{-1}$ in region I and $\beta_{z 2}=1.3492 * 10^{7} \mathrm{~m}^{-1}$ in region II. The propagation constants are evaluated by considering the dispersion equation for a multilayer dielectric structure and through the effective dielectric constant method [12, 17]. In this case the phase velocity and the group velocity are near the asymptotic condition $n_{2} k_{0}=\beta_{z}$ (see Figure 3 ). The Brillouin diagram of the $3 \mathrm{D}$ GaAs periodic structure is reported in Figure 4 in which two different periodicities are considered along the $y$ - and $z$-axis. Moreover Figure 5 shows the Brillouin diagram of the same GaAs structure in the case of same periodicity along the $y$-and $z$-axis. Before to analyze the transmittivity and Floquet's resonance condition some aspects of the theoretical model are illustrated: in particular Figure 6 reports how Floquet's condition (6) changes with the ratio transformation $r$ by fixing $L_{1}$ and the resonant wavelength $\lambda_{0}$ (more choices of $L_{2}$ are possible with low ratio transformation); moreover the effect of the cell number on the transition bands is shown in Figure 7 where the transmittivity responses versus $\theta$ for different unit cell number are considered. In order to validate our model for a multimode analysis a multimode $2 \mathrm{D}$ periodic slab waveguide with bandpass centred in $\lambda_{0}=1.55 \mu \mathrm{m}$ is considered. Regarding this example the same periodicity along the $y$ $z$-propagation directions and the wavenumber conservation law of (11) are considered. By taking into account the effective refractive indices and (22), the $2 \mathrm{D}$ case becomes a simplified case of the $3 \mathrm{D}$ case. The Brillouin diagram of Figure 5 shows the propagation conditions related to this example. For a resonant central wavelength of $\lambda_{0}=1.55 \mu \mathrm{m}$, a possible geometrical configuration is the unit cell (along the periodic $y z$ plane) with $L_{1}=0.1163 \mu \mathrm{m}, L_{2}=0.1246 \mu \mathrm{m}$. By (21) a ratio transformation of $r=5$ is calculated by considering $n_{2}=3.408, n_{1}=1, n_{3}=3.1, d=0.24 \mu \mathrm{m}$, $D=0.8 \mu \mathrm{m}$ and all the TE propagating modes in the GaAs slab with core thickness $d$ and $D$ (in particular one TE propagating mode in the region with thickness $d$ and two TE propagating modes in the region with thickness $D$ are considered). Figures 8 and 9 show the transmittivity and Floquet's conditions for this example. Moreover Figure 9(b) indicates that the limit points A and B of Floquet's condition remain fixed by increasing the unit cell number; therefore, the single unit cell gives information about the passband centred at the resonance wavelength $\lambda_{0}$. In Figures 10(a), $10(\mathrm{~b})$, and 10 (c) is reported how it is possible to localize

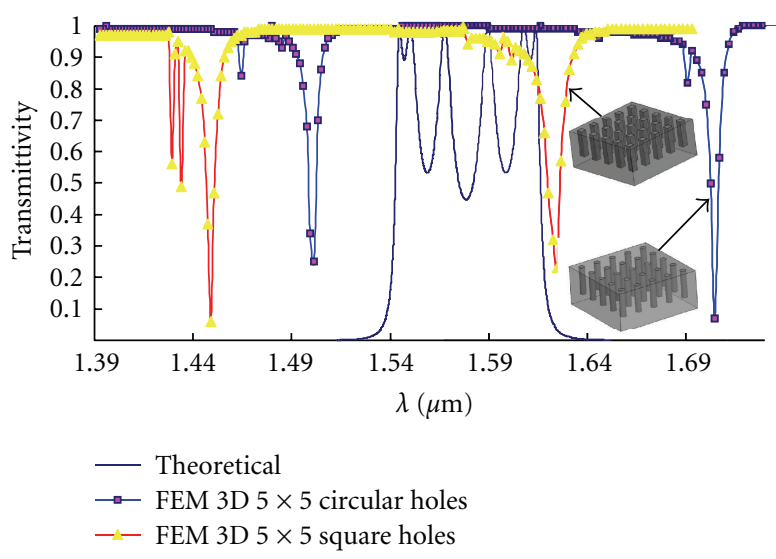

FIGURE 11: Transmittivity response of the 2D theoretical and 3D FEM modeling.

Floquet's region for five unit cells: Floquet's region of the single cell is superimposed to the transmission characteristic of the periodic structure by defining the passband condition.

\section{Bandpass Optimization: FEM Modeling}

After the design of the 2D periodic structure we optimize the periodic structure by analyzing the diffraction effect on the frequency response. The different air hole geometries and the air hole depths may change the bandwidth and the cutoff frequencies position. The full wave FEM method considers all these aspects also for complex $3 \mathrm{D}$ periodic structures with high unit cells number. The numerical modelling optimizes the bandpass position around the theoretical prediction.

In Figure 11 is presented the comparison of the passband between the theoretical model and the 3D FEM simulation: the theoretical model takes into account the same periodicity along the $y$ - and $z$-axis and the Brillouin diagram of Figure 5. Also in the 3D FEM simulation a passband around $\lambda_{0}=$ $1.55 \mu \mathrm{m}$ is checked. The difference of the bandwidth and the different cutoff frequencies with respect to the theoretical model is in the particular geometry of the periodic pattern (scattering effects of circular and square air holes with a defined depth). Figure 11 shows that the 2D theoretical passband is always centred, and that the $3 \mathrm{D}$ model is useful in order to increase the bandwidth or to shift the cutoff frequencies (optimization process). In the FEM simulator the excitations are wave ports placed at the boundary interface in order to provide a window (cross sections) that couples the model device to the external space. The electromagnetic simulator assumes that the structure is excited by the natural field patterns (TE mode profiles according to the theoretical hypothesis) associated with these cross-sections. The $2 \mathrm{D}$ field solution generated for each wave port is the exact modal boundary condition at those ports for the 3D problem. Figure 12 proves the $3 \mathrm{D}$ resonance at $\lambda_{0}=1.55 \mu \mathrm{m}$ by considering the unit cell dimensions of the $2 \mathrm{D}$ theoretical model (same unit cells along the $y$ - and $z$-direction). 


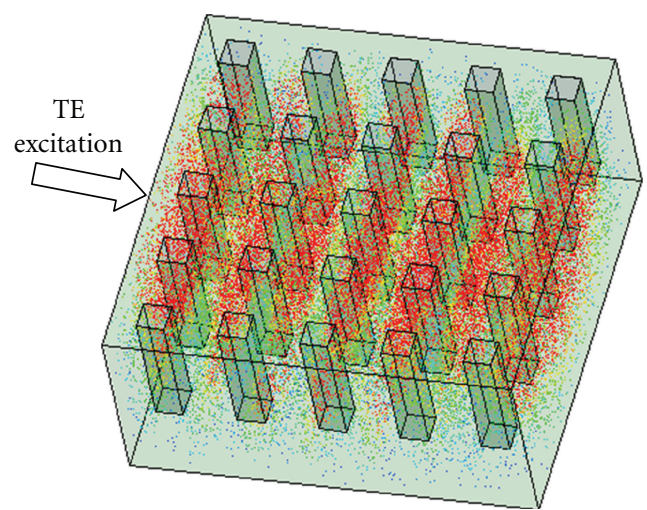

(a)

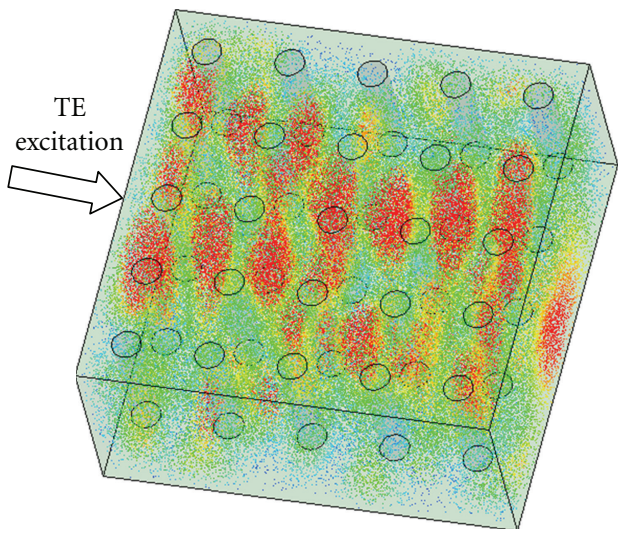

(c)

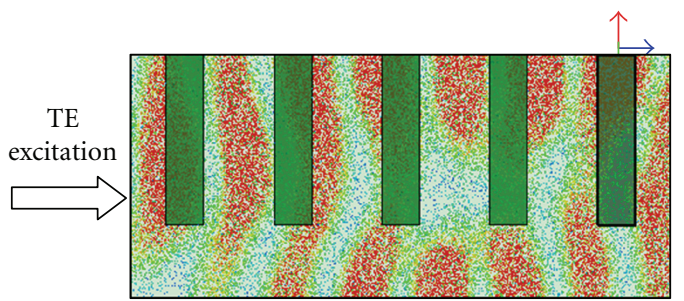

(b)

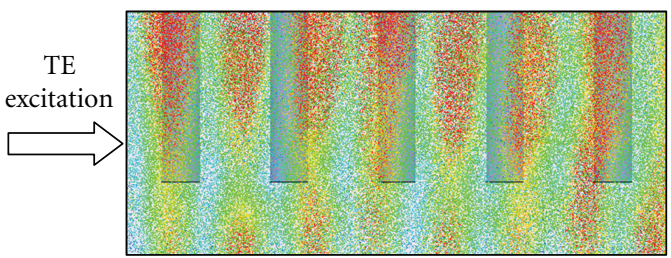

(d)

FIGURE 12: Volumetric electric field distribution of $5 \times 5$ unit cells with $L_{1}=0.1163 \mu \mathrm{m}, L_{2}=0.1246 \mu \mathrm{m}$ : (a) top view of $5 \times 5$ square unit cells, (b) longitudinal section of $5 \times 5$ square unit cells, (c) top view of $5 \times 5$ circular unit cells, and (d) longitudinal section of $5 \times 5$ circular unit cells.

\section{Conclusion}

To summarize we presented in this work a direct and accurate theoretical approach regarding the resonance frequencies design of dielectric periodic structures with high dielectric contrast. The rigorous method is based on Floquet's transmission line theory and on the equivalent variational circuit which models the discontinuity effect on the frequency response at optical frequencies. The design is performed by the Brillouin diagram which defines, through the wavenumber conservation law, the unit cell dimension of the resonating structure. The 3D FEM simulation verifies the resonance of a GaAs photonic crystal with air holes obtained by the theoretical model around a working wavelength of $\lambda_{0}=$ $1.55 \mu \mathrm{m}$. The FEM numerical tool optimizes the bandwidth and the cutoff frequencies position by considering different air hole geometries. The model can be also applied to design photonic band gap devices with microcavities.

\section{References}

[1] S. Noda, A. Chutinan, and M. Imada, "Trapping and emission of photons by a single defect in a photonic bandgap structure," Nature, vol. 407, pp. 608-610, 2000.
[2] M. Imada, S. Noda, A. Chutinan, T. Tokuda, M. Murata, and G. Sasaki, "Coherent two-dimensional lasing action in surface-emitting laser with triangular-lattice photonic crystal structure," Applied Physics Letters, vol. 75, no. 3, pp. 316-318, 1999.

[3] S. Noda, M. Yokoyama, M. Imada, A. Chutinan, and M. Mochizuki, "Polarization mode control of two-dimensional photonic crystal laser by unit cell structure design," Science, vol. 293, no. 5532, pp. 1123-1125, 2001.

[4] S. Fan, P. R. Villneuve, M. Joannopoulos, and J. D. Haus, "Channel drop tunneling througth localized states," Physical Review Letters, vol. 80, pp. 960-963, 1998.

[5] C. Manolatou, M. J. Khan, S. Fan, P. R. Villeneuve, H. A. Haus, and J. D. Joannopoulos, "Coupling of modes analysis of resonant channel add-drop filters," IEEE Journal of Quantum Electronics, vol. 35, no. 9, pp. 1322-1331, 1999.

[6] M. Bayindir and E. Ozbay, "Band-dropping via coupled photonic crystal waveguides," Optics Express, vol. 10, no. 22, pp. 1279-1283, 2002.

[7] M. Qiu, "Ultra-compact optical filter in two-dimensional photonic crystal," Electronics Letters, vol. 40, no. 9, pp. 539540, 2004.

[8] M. Qiu and B. Jaskorzynska, "Design of a channel drop filter in a two-dimensional triangular photonic crystal," Applied Physics Letters, vol. 83, no. 6, pp. 1074-1076, 2003. 
[9] A. D’Orazio, M. De Sario, V. Petruzzelli, and F. Prudenzano, "Photonic band gap filter for wavelength division multiplexer," Optics Express, vol. 11, no. 3, pp. 230-239, 2003.

[10] M. Koshiba, "Wavelength division multiplexing and demultiplexing with photonic crystal waveguide couplers," Journal of Lightwave Technology, vol. 19, no. 12, pp. 1970-1975, 2001.

[11] A. Sharkawy, S. Shi, and D. W. Prather, "Multichannel wavelength division multiplexing with photonic crystals," Applied Optics, vol. 40, no. 14, pp. 2247-2252, 2001.

[12] T. Rozzi and M. Mongiardo, Open Electromagnetic Waveguides, vol. 43 of IEE Electromagnetic Waves Series, chapters 3, 4, 5, The Institute of Electrical Engineering, London, UK, 1997.

[13] C. A. Olley, T. Rozzi, and C. M. D. Rycroft, "An approximate variational solution to the step discontinuity in finline," IEEE Transactions on Microwave Theory and Techniques, vol. 37, no. 6, pp. 977-983, 1989.

[14] R. E. Collin, Foundation for Microwave Engineering, McGrawHill, New York, NY, USA, 2nd edition, 1992.

[15] D. M. Pozar, Microwave Engineering, John Wiley \& Sons, New York, NY, USA, 2nd edition, 1998.

[16] A. Massaro, L. Pierantoni, and T. Rozzi, "Resonances of 2D mesa periodic structures in integrated optics 3 by unit cell structure design," Microwave and Optical Technology Letters, vol. 48, no. 4, pp. 629-632, 2006.

[17] L. Pierantoni, A. Massaro, and T. Rozzi, "Accurate modeling of TE/TM propagation and losses of integrated optical polarizer," IEEE Transactions on Microwave Theory and Techniques, vol. 53, no. 6, part 1, pp. 1856-1862, 2005.

[18] S. Boscolo, C. Conti, M. Midrio, and C. G. Someda, "Numerical analysis of propagation and impedance matching in 2D photonic crystal waveguides with finite length," Journal of Lightwave Technology, vol. 20, no. 2, pp. 304-310, 2002.

[19] J. Zarbakhsh, A. Mohtashami, K. Hingerl, L. Tkeshelashvili, and K. Busch, "Improving the impedance matching in photonic crystal waveguides," in Proceedings of the International Conference on Transparent Optical Networks (ICTON '06), vol. 4 , p. $275,2006$. 

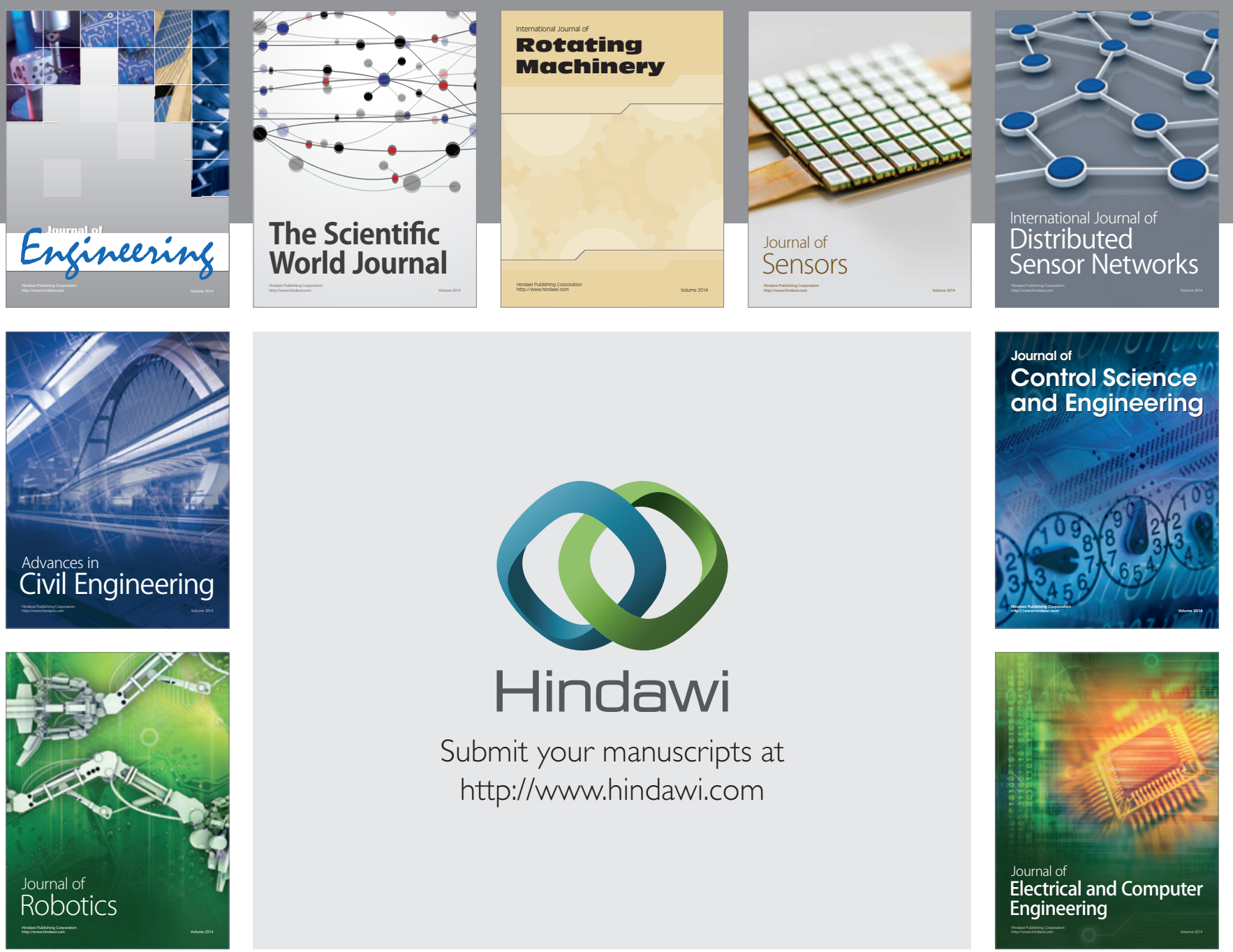

Submit your manuscripts at

http://www.hindawi.com
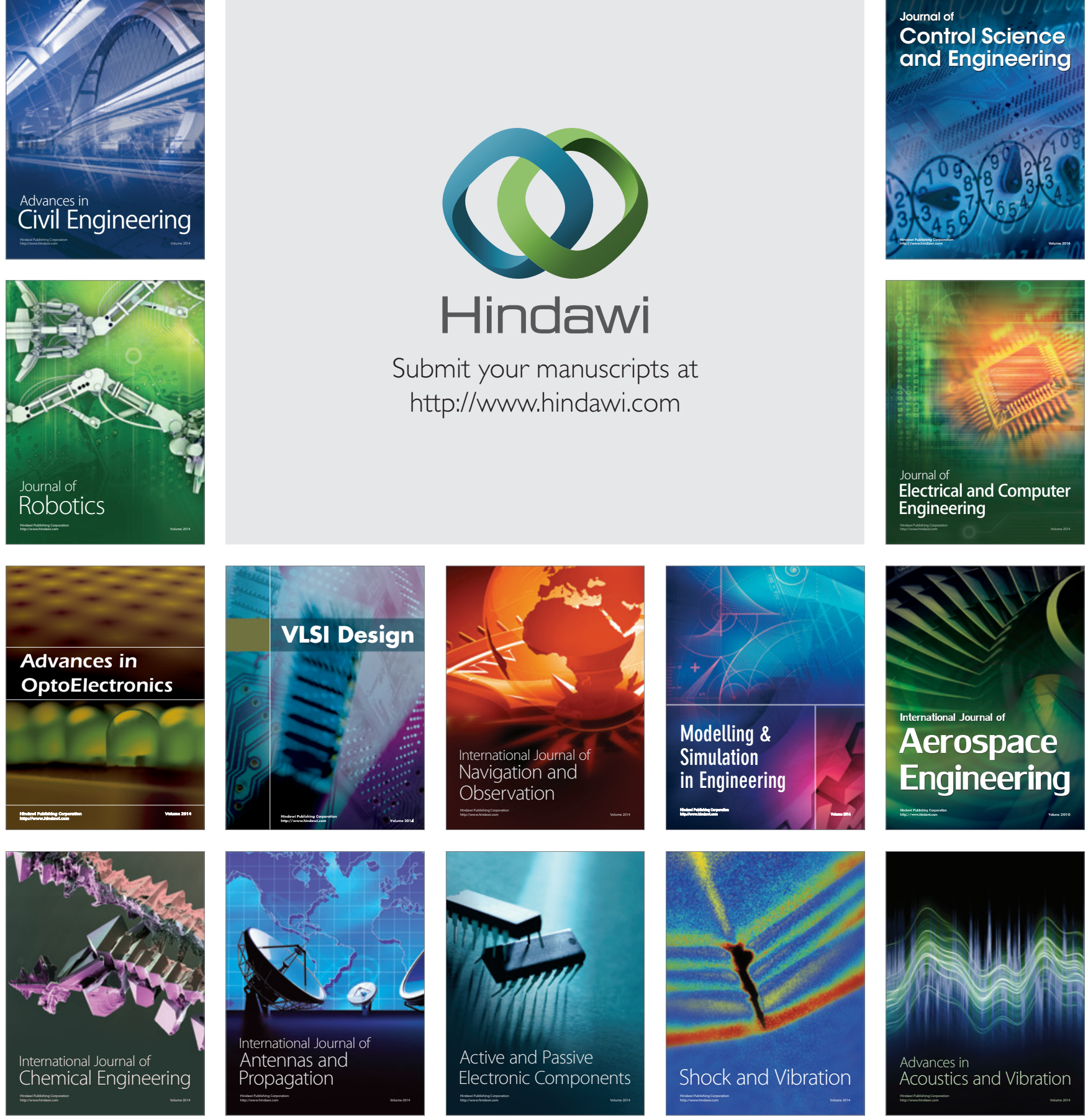